\title{
Trimethoprim-sulfamethoxazole induced hyperkalaemia in elderly patients receiving spironolactone: nested case-control study
}

\author{
(c) $(1)$ (8)
}

\author{
Tony Antoniou clinical pharmacy specialist ${ }^{12}$, Tara Gomes epidemiologist ${ }^{13}$, Muhammad M Mamdani \\ director $^{1345}$, Zhan Yao analyst ${ }^{3}$, Chelsea Hellings project manager ${ }^{3}$, Amit X Garg professor ${ }^{36}$, \\ Matthew A Weir assistant professor of medicine ${ }^{6}$, David N Juurlink division head ${ }^{137}$
}

${ }^{1}$ University of Toronto, Toronto, ON, Canada ; ${ }^{2}$ Department of Family and Community Medicine, St Michael's Hospital, Toronto ; ${ }^{3}$ Institute for Clinical Evaluative Sciences, Toronto; ${ }^{4} \mathrm{Li}$ Ka Shing Knowledge Institute, St Michael's Hospital, Toronto ; ${ }^{5}$ King Saud University, Riyadh, Saudi Arabia; ${ }^{6}$ Division of Nephrology, University of Western Ontario, London, ON, Canada ; ${ }^{7}$ Sunnybrook Research Institute, Toronto

\begin{abstract}
Objectives To characterise the risk of admission to hospital for hyperkalaemia in elderly patients treated with trimethoprim-sulfamethoxazole in combination with spironolactone. Design Population based nested case-control study.

Setting Ontario, Canada, from 1 April 1992 to 1 March 2010.

Participants Cases were residents of Ontario aged 66 years or above receiving chronic treatment with spironolactone and admitted to hospital with hyperkalaemia within 14 days of receiving a prescription for either trimethoprim-sulfamethoxazole, amoxicillin, norfloxacin, or nitrofurantoin. Up to four controls for each case were identified from the same cohort, matched on age, sex, and presence or absence of chronic kidney disease and diabetes, and required to have received one of the study antibiotics within 14 days before the case's index date.
\end{abstract}

Main outcome measures Odds ratio for association between admission to hospital with hyperkalaemia and receipt of a study antibiotic in the preceding 14 days, adjusted for conditions and drugs that may influence risk of hyperkalaemia.

Results During the 18 year study period, 6903 admissions for hyperkalaemia were identified, 306 of which occurred within 14 days of antibiotic use. Of these, $248(81 \%)$ cases were matched to 783 controls. $10.8 \%$ (17 859/165 754) of spironolactone users received at least one prescription for trimethoprim-sulfamethoxazole. Compared with amoxicillin, prescription of trimethoprim-sulfamethoxazole was associated with a marked increase in the risk of admission to hospital for hyperkalaemia (adjusted odds ratio 12.4, 95\% confidence interval 7.1 to 21.6 ). The population attributable fraction was $59.7 \%$, suggesting that approximately $60 \%$ of all cases of hyperkalaemia in older patients taking spironolactone and treated with an antibiotic for a urinary tract infection could be avoided if trimethoprim-sulfamethoxazole was not prescribed. Treatment with nitrofurantoin was also associated with an increase in the risk of hyperkalaemia (adjusted odds ratio $2.4,1.3$ to 4.6 ), but no such risk was found with norfloxacin (adjusted odds ratio 1.6, 0.8 to 3.4)

Conclusions Among older patients receiving spironolactone, treatment with trimethoprim-sulfamethoxazole was associated with a major increase in the risk of admission to hospital for hyperkalaemia. This drug combination should be avoided when possible.

\section{Introduction}

Spironolactone is a non-selective mineralocorticoid receptor antagonist used to treat systolic heart failure, resistant hypertension, ascites, and hyperaldosteronism..$^{1-3}$ The prescribing of spironolactone increased considerably after the publication of the Randomized Aldactone Evaluation Study (RALES), which showed that the drug could reduce morbidity and mortality in patients with severe left ventricular dysfunction. ${ }^{4-6}$ Although spironolactone was generally well tolerated in that study, serious hyperkalaemia is an important consideration for patients receiving the drug in clinical practice. Subsequent research has shown that hyperkalaemia occurs in up to a third of patients receiving spironolactone, emphasising the need for regular monitoring of electrolytes and the avoidance of other drugs that can cause hyperkalaemia. ${ }^{7-9}$

The antibiotic trimethoprim has structural and pharmacological similarities to the potassium sparing diuretic amiloride and reduces urinary potassium excretion by approximately $40 \% .^{10} 11$ The inhibition of potassium secretion results in a dose related antikaliuretic effect that may predispose susceptible people to clinically important hyperkalaemia. ${ }^{12}$ In combination with 
sulfamethoxazole, trimethoprim is commonly used for the treatment of urinary tract infections. Because trimethoprim-sulfamethoxazole and spironolactone are both widely used drugs, the likelihood of co-prescription is high. However, the risk of hyperkalaemia associated with the combined use of these drugs has not been systematically studied, and evidence supporting a clinically meaningful drug interaction is limited to case reports. ${ }^{13}{ }^{14} \mathrm{We}$ therefore sought to characterise the significance of this drug interaction in clinical practice.

\section{Methods}

We did a population based, nested case-control study of Ontario residents aged 66 years or older treated with spironolactone between 1 April 1992 and 1 March 2010. We determined prescription drug use by using the Ontario Drug Benefit Database, which identifies prescriptions dispensed to Ontario residents aged 65 years or older. Hospital admission data came from the Canadian Institute for Health Information's Discharge Abstract Database and demographic information from the Registered Persons Database. The Discharge Abstract Database contains clinical information on all admissions, discharges, and same day surgeries from all hospitals in Ontario. Trained health information professionals abstract patients' charts by using standard diagnosis and procedure codes. We used the Ontario Health Insurance Plan database to identify claims for physicians' services and the Ontario Diabetes Database for information on diagnoses of diabetes. ${ }^{15}$ These databases were linked in an anonymous fashion by using encrypted health card numbers and are regularly used to study drug safety, including the consequences of drug interactions. ${ }^{516-18}$

For each patient, we identified a period of continuous use of spironolactone beginning with the first prescription for spironolactone after the patient's 66th birthday. We excluded the first year of eligibility for coverage of prescription drugs (age 65) to avoid incomplete drug records. Observation ended with the first occurrence of a hospital admission for hyperkalaemia, death, the end of the study period (31 March 2010), or cessation of spironolactone treatment, defined as a lapse of more than 180 days between prescriptions. In the event of such a lapse, we extended the observation period 180 days from the date of the last prescription to identify outcomes that may have precipitated cessation of treatment.

Within the cohort of continuous users of spironolactone, we defined cases as those admitted to hospital with a diagnosis of hyperkalaemia (international classifications of diseases, 9th edition, code 276.7, and 10th edition, code E87.5) within 14 days of receiving a prescription for one of four study antibiotics: trimethoprim-sulfamethoxazole, norfloxacin, nitrofurantoin, or amoxicillin. We did not include prescriptions for trimethoprim monotherapy, because the drug is invariably used in combination with sulfamethoxazole in Canada. We included only patients who had hyperkalaemia at the time of admission, rather than those who developed hyperkalaemia during the course of a hospital admission. We restricted our analyses to antibiotics that are primarily used to treat urinary tract infections to avoid the potential confounding effects of other systemic infections. The date of hospital admission served as the index date for all analyses, and we considered only the first instance of hospital admission for hyperkalaemia for patients with more than one such admission during the study period. We excluded patients if they received prescriptions for multiple antibiotics or any non-study antibiotics in the 30 days before the index date.

From within the cohort of patients receiving spironolactone, we selected up to four controls for each case by using incidence density sampling. ${ }^{19}$ We required controls to have had no hospital admission for hyperkalaemia before the index date and to have received one of the study antibiotics within 14 days before the index date. Consequently, all cases and controls were older patients receiving spironolactone who had also received treatment with one of the study antibiotics. We matched controls and cases on age at the index date (plus or minus one year), sex, presence or absence of diabetes (based on review of the validated Ontario Diabetes Database ${ }^{15}$ ), and presence or absence of chronic kidney disease, determined from physicians' claims, hospital admission records, and receipt of dialysis in the year before the index date. Each patient could serve only once as a control. When fewer than four control patients were available for each case, we analysed only those controls and maintained the matching process. We excluded any cases that could not be matched to at least one control.

\section{Statistical analysis}

We calculated descriptive statistics for baseline demographic and clinical characteristics of cases and controls, as well as standardised differences to test for differences between the two groups. Standardised differences of less than 0.1 indicate a good balance between the cases and controls for a given covariate. ${ }^{20}$

We used conditional logistic regression to estimate the odds ratio and $95 \%$ confidence intervals for the association between hyperkalaemia related hospital admission and receipt of a prescription for trimethoprim-sulfamethoxazole in the preceding 14 days, using amoxicillin treated patients as the reference group. We selected amoxicillin as the reference antibiotic because, with rare exceptions, it should not cause hyperkalaemia. ${ }^{21}$ To test the specificity of our findings, we also examined the association between hyperkalaemia and prescription of nitrofurantoin or norfloxacin, antibiotics commonly used to treat community acquired urinary tract infections. We hypothesised that these drugs would not be associated with an increased risk of hyperkalaemia relative to amoxicillin. We used multivariable conditional logistic regression to adjust for medical conditions and classes of prescription drugs that may influence the risk of hyperkalaemia (see web appendix). ${ }^{22-25}$ To ascertain whether a dose-response relation existed between trimethoprim and hyperkalaemia, we repeated our primary analysis, stratifying exposure to trimethoprim-sulfamethoxazole according to prescription for single strength $(400 \mathrm{mg} / 80 \mathrm{mg})$ or double strength $(800 \mathrm{mg} / 160$ $\mathrm{mg}$ ) tablets. Finally, we determined the population attributable fraction, defined in this study as the fraction of all cases (exposed and unexposed) that would not have occurred if exposure to trimethoprim-sulfamethoxazole had been avoided. ${ }^{26}$ We used SAS version 9.2 for all analyses.

\section{Results}

We identified 165754 patients treated with spironolactone during the 18 year study period, of which 17859 (10.8\%) received at least one prescription for trimethoprim-sulfamethoxazole during the study period. Within the cohort of spironolactone users, we identified 6903 admissions for hyperkalaemia, 306 of which occurred within 14 days of receiving an antibiotic of interest. Most cases ( $\mathrm{n}=248$; $81 \%$ ) were matched to at least one control. Cases and controls were similar with respect to age, residence in a long term care facility, and history of diabetes (table $1 \Downarrow$ ). As expected, comorbidities and drugs known to increase the risk of hyperkalaemia were more prevalent among cases than among controls. 
In the primary analysis, trimethoprim-sulfamethoxazole was associated with a marked increase in the risk of hospital admission for hyperkalaemia among patients receiving spironolactone (table $2 \Downarrow$ ). After multivariable adjustment, patients admitted to hospital with hyperkalaemia were more than 12 times as likely to have received a recent prescription for trimethoprim-sulfamethoxazole than for amoxicillin (adjusted odds ratio 12.4, 95\% confidence interval 7.1 to 21.6). When we stratified the data by dose, we found a higher risk of hospital admission for hyperkalaemia with the double strength formulation of trimethoprim-sulfamethoxazole (adjusted odds ratio $13.5,7.5$ to 24.2 ) than with single strength tablets (adjusted odds ratio 9.7, 4.7 to 19.9). Treatment with nitrofurantoin was also associated with an increased risk of hyperkalaemia during spironolactone treatment (adjusted odds ratio $2.4,1.3$ to 4.6). In contrast, we found no association between hyperkalaemia and receipt of norfloxacin (adjusted odds ratio 1.6, 0.8 to 3.4).

The population attributable fraction was $59.7 \%$, suggesting that approximately $60 \%$ of all cases of hyperkalaemia in older patients taking spironolactone and treated with an antibiotic for a urinary tract infection could be avoided if trimethoprim-sulfamethoxazole was not prescribed.

Finally, because we did not attain a full match for all of our cases, we did a sensitivity analysis matching cases and controls only on sex, thereby matching all cases to at least one control. Results of the sensitivity analysis were consistent with those obtained with the originally matched cohort.

\section{Discussion}

In this population based study spanning 18 years, we found that the prescription of trimethoprim-sulfamethoxazole was strongly associated with admission to hospital for hyperkalaemia among elderly patients treated with spironolactone. Treatment with nitrofurantoin was associated with a less pronounced increase in the risk of hospital admission for hyperkalaemia, whereas we found no such risk with norfloxacin. Our findings support the existence of a clinically important and potentially life threatening drug interaction between

trimethoprim-sulfamethoxazole and spironolactone at the population level. This represents the first estimate of the risk of co-prescribing these drugs in clinical practice.

We found a less striking increase in the risk of hyperkalaemia with nitrofurantoin treatment. This may reflect a "channelling" effect, in which patients at greater risk of hyperkalaemia were more likely to have been prescribed nitrofurantoin rather than trimethoprim-sulfamethoxazole, although this same phenomenon should also apply to norfloxacin. Some evidence indicates that this association may reflect a causal relation. In vitro experiments show that nitrofurantoin inhibits steroid biosynthesis, resulting in a 50\% reduction in aldosterone release from adrenocortical cells. ${ }^{28}$ An additional, previously underappreciated drug interaction may thus exist between nitrofurantoin and spironolactone that may predispose susceptible patients to hyperkalaemia.

Our study has important clinical implications. In our cohort, $10.8 \%$ of patients received at least one prescription for trimethoprim-sulfamethoxazole during the study period and were therefore at increased risk of hyperkalaemia. Serious hyperkalaemia can cause sudden death and is the most dangerous complication of treatment with spironolactone. Consequently, strategies aimed at minimising the risk of hyperkalaemia are essential. Our findings suggest that in patients receiving spironolactone, avoidance of trimethoprim-sulfamethoxazole is prudent when clinically appropriate.

\section{Limitations}

Some limitations of our work merit emphasis. We used administrative data and had no access to serum potassium concentrations, indices of renal function (including estimated glomerular filtration rate), adherence to drug treatment, or use of non-prescription drugs that may have influenced the risk of hyperkalaemia. However, these limitations apply equally to all antibiotics studied, and in particular would not be expected to differ appreciably between trimethoprim-sulfamethoxazole and amoxicillin. We also could not identify hyperkalaemia in outpatients and hyperkalaemia related mortality in the pre-hospital setting. However, this would lead to underestimation of the clinical consequences of this drug interaction. Because some clinicians may appreciate the risk of hyperkalaemia with trimethoprim-sulfamethoxazole, differential outcome ascertainment is a possible source of bias. However, this is unlikely to have affected our findings as electrolyte concentrations are routinely measured in older patients presenting to hospital. In addition, our findings may not apply to younger patients with fewer risk factors for hyperkalaemia. Furthermore, as with all observational studies, residual confounding is a potential source of bias. However, given the magnitude of the observed association, we find it difficult to conceive of an unmeasured confounder that would be differentially distributed among the various antibiotics that could be accounting for our findings. Finally, the accuracy of hospital discharge coding for hyperkalaemia has not been validated, although the same limitation applies to previous research on the clinical consequences of drug interactions leading to hyperkalaemia. ${ }^{516}$

\section{Conclusions}

Prescription of trimethoprim-sulfamethoxazole to elderly patients receiving treatment with spironolactone was associated with a major increase in the risk of hyperkalaemia, and this risk greatly exceeded that observed with other commonly used antibiotics for urinary tract infection. Increased awareness of this drug interaction among pharmacists and physicians is needed to ensure that the potential for life threatening hyperkalaemia with this drug combination is minimised, either by selection of alternative antibiotics when appropriate or by close monitoring of patients treated with both drugs.

We thank Brogan, Ottawa, for use of their Drug Product and Therapeutic Class Database.

Contributors: TA, TG, DNJ, MMM, CH, AXG, and MAW were involved in the study concept and design. TG and $Z Y$ were responsible for data acquisition. All authors were involved in analysing and interpreting data. TA drafted the manuscript, and all authors critically revised it. TA, TG, and $\mathrm{CH}$ provided administrative, technical, or material support. TA is the guarantor.

Funding: TA is supported by a post-doctoral fellowship award from the Ontario HIV Treatment Network. AG is supported by a clinician scientist award from the Canadian Institutes of Health Research. This project was supported by research funds from the Ontario Drug Policy Research Network and by the Institute for Clinical Evaluative Sciences (ICES), which is funded by an annual grant from the Ontario Ministry of Health and Long-Term Care (MOHLTC). The sponsors had no role in the design and conduct of the study; in the collection, analysis, and interpretation of the data; or in the preparation, review, or approval of the manuscript. The opinions, results, and conclusions reported in this paper are those of the authors and are independent from the funding sources. No endorsement by ICES or the Ontario MOHLTC is intended or should be inferred 


\section{What is already known on this topic}

Drug interactions are an important and avoidable cause of hyperkalaemia

Trimethoprim (generally combined with sulfamethoxazole in North America) and spironolactone can both cause hyperkalaemia, yet the risks associated with this combination are unknown

\section{What this study adds}

Compared with amoxicillin, prescription of trimethoprim-sulfamethoxazole was associated with a more than 12 -fold increase in the risk of hospital admission for hyperkalaemia among older patients taking spironolactone

$10.8 \%$ of spironolactone users received at least one prescription for trimethoprim-sulfamethoxazole, thereby being placed at excess risk of hyperkalaemia

Competing interests: All authors have completed the Unified Competing Interest form at www.icmje.org_disclosure.pdf (available on request from the corresponding author). During the past three years, TA has received unrestricted research grants from GlaxoSmithKline, Merck, and Pfizer for different studies. All other authors declare no support from any company for the submitted work; no relationships with any companies that might have an interest in the submitted work in the previous 3 years; and no non-financial interests that may be relevant to the submitted work.

Ethical approval: This study was approved by the Research Ethics Board of the Sunnybrook Health Sciences Centre, Toronto.

Data sharing: No additional data available.

1 Rocha R, Williams GH. Rationale for the use of aldosterone antagonists in congestive heart failure. Drugs 2002;62:723-31.

2 Schrier RW, Masoumi A, Elhassan E. Aldosterone: role in edematous disorders, hypertension, chronic renal failure and metabolic syndrome. Clin J Am Soc Nephrol 2010:5:1132-40

3 Lim PO, Young WF, MacDonald TM. A review of the medical treatment of primary aldosteronism. $J$ Hypertens 2001;19:353-61.

4 Pitt B, Zannad F, Remme WJ, Cody R, Castaigne A, Perez A, et al. The effect of spironolactone on morbidity and mortality in patients with severe heart failure. $N$ Engl $J$ Med 1999;341:709-17.

5 Juurlink DN, Mamdani MM, Lee DS, Kopp A, Austin PC, Laupacis A, et al. Rates of hyperkalemia after publication of the Randomized Aldactone Evaluation Study. N Engl J Med 2004;351:543-51.

6 Masoudi FA, Gross CP, Wang Y, Rathore SS, Havranek EP, Foody JM, et al. Adoption of spironolactone therapy for older patients with heart failure and left ventricular systolic dysfunction in the United States, 1998-2001. Circulation 2005;112:39-47.

7 Bozkurt B, Agoston I, Knowlton AA. Complications of inappropriate use of spironolactone in heart failure: when an old medicine spirals out of new guidelines. J Am Coll Cardiol 2003:41:211-4.

8 Svensson M, Gustafsson F, Galatius S, Hildebrandt PR, Atar D. How prevalent is hyperkalemia and renal dysfunction during treatment with spironolactone in patients with congestive heart failure? J Card Fail 2004;10:297-303.

9 Cruz CS, Cruz AA, Marcílio de Souza CA. Hyperkalemia in congestive heart failure patients using ACE inhibitors and spironolactone. Nephrol Dial Transplant 2003:18:1814-9.

10 Eiam-ong S, Kurtzman NA, Sabatini S. Studies on the mechanism of trimethoprim-induced hyperkalemia. Kidney Int 1996;49:1372-8.

11 Velazquez H, Perazella MA, Wright FS, Ellison DH. Renal mechanism of trimethoprim-induced hyperkalemia. Ann Intern Med 1993;119:296-301.

12 Perazella MA. Trimethoprim-induced hyperkalemia: clinical data, mechanism, prevention and management. Drug Saf 2000;22:227-36.
13 Marinella MA. Severe hyperkalemia associated with trimethoprim-sulfamethoxazole and spironolactone. Infect Dis Clin Pract 1997;6:257.

14 Martin J, Mourton S, Nicholls G. Severe hyperkalemia with prescription of potassium-retaining agents in an elderly patient. N Z Med J 2003;116:U542.

15 Hux JE, Ivis F, Flintoft V, Bica A. Diabetes in Ontario: determination of prevalence and incidence using a validated administrative data algorithm. Diabetes Care 2002;25:512-6.

16 Juurlink DN, Mamdani M, Kopp A, Laupacis A, Redelmeier DA. Drug-drug interactions among elderly patients hospitalized for drug toxicity. JAMA 2003;289:1652-8.

17 Park-Wyllie LY, Juurlink DN, Kopp A, Shah BR, Stukel TA, Stumpo C, et al. Outpatient gatifloxacin therapy and dysglycemia in older adults. N Engl J Med 2006;354:1352-61.

18 Mamdani M, Juurlink DN, Lee DS, Rochon PA, Kopp A, Naglie G, et al. Cyclo-oxygenase-2 inhibitors versus non-selective non-steroidal anti-inflammatory drugs and congestive heart failure outcomes in elderly patients: a population-based cohort study. Lancet 2004:363:1751-6.

19 Lubin JH, Gail MH. Biased selection of controls for case-control analyses of cohort studies. Biometrics 1984;40:63-75.

20 Austin PC, Grootendorst P, Anderson GM. A comparison of the ability of different propensity score models to balance measured variables between treated and untreated subjects: a Monte Carlo study. Stat Med 2007;26:734-53.

21 Appel GB, Garvey G, Silva F, Francke E, Neu HC, Weissman J. Acute interstitial nephritis due to amoxicillin therapy. Nephron 1981;27:313-5.

22 Desai AS, Swedberg K, McMurray JJ, Granger CB, Yusuf S, Young JB, et al. Incidence and predictors of hyperkalemia in patients with heart failure: an analysis of the CHARM program. J Am Coll Cardiol 2007;50:1959-66.

23 Ponce SP, Jennings AE, Madias NE, Harrington JT. Drug-induced hyperkalemia. Medicine (Baltimore) 1985;64:357-70.

24 Charlson ME, Pompei P. Ales KL, MacKenzie CR. A new method of classifying prognostic comorbidity in longitudinal studies: development and validation. $J$ Chronic Dis 1987; 40:373-83.

25 Schneeweiss S, Seeger JD, Maclure M, Wang PS, Avorn J, Glynn RJ. Performance of comorbidity scores to control for confounding in epidemiologic studies using claims data. Am J Epidemiol 2001;154:854-64.

26 Hanley JA. A heuristic approach to the formulas for population attributable fraction. $J$ Epidemiol Community Health 2001;55:508-14.

27 Rockhill B, Newman B, Weinberg C. Use and misuse of population attributable fractions. Am J Public Health 1998;17:15-9.

28 Jager LP, de Graaf GJ, Widjaja-Greefkes HCA. Differential effects of nitrofurans on the production/release of steroid hormones by porcine adrenocortical cells in vitro. Eur $J$ Pharmacol 1997;331:325-31.

Accepted: 22 July 2011

\section{Cite this as: BMJ 2011;343:d5228}

This is an open-access article distributed under the terms of the Creative Commons Attribution Non-commercial License, which permits use, distribution, and reproduction in any medium, provided the original work is properly cited, the use is non commercial and is otherwise in compliance with the license. See: http://creativecommons.org/licenses/bync/2.0/ and http://creativecommons.org/licenses/by-nc/2.0/legalcode. 


\section{Tables}

\section{Table 1| Characteristics of cases and controls. Values are numbers (percentages) unless stated otherwise}

\begin{tabular}{|c|c|c|c|}
\hline Characteristics & Cases $(n=248)$ & Controls ( $n=783$ ) & Standardised difference ${ }^{*}$ \\
\hline Median (interquartile range) age (years): & $82(75-87)$ & $81(75-87)$ & 0.01 \\
\hline $66-74$ & $66(27)$ & $203(26)$ & 0.02 \\
\hline $75-84$ & $112(45)$ & $370(47)$ & 0.04 \\
\hline$\geq 85$ & $70(28)$ & $210(27)$ & 0.03 \\
\hline Female sex & $162(65)$ & $551(70)$ & 0.11 \\
\hline Median (interquartile range) years of spironolactone use & $1(1-2)$ & $3(1-5)$ & 0.66 \\
\hline \multicolumn{4}{|l|}{ Charlson co-morbidity index: } \\
\hline No hospital admission & $37(15)$ & $214(27)$ & 0.29 \\
\hline 0 & $13(5)$ & $94(12)$ & 0.22 \\
\hline 1 & $41(17)$ & $131(17)$ & 0.01 \\
\hline$\geq 2$ & $157(63)$ & $344(44)$ & 0.39 \\
\hline History of congestive heart failure (3 years) & $132(53)$ & $282(36)$ & 0.35 \\
\hline History of chronic liver disease (1 year) & $26(10)$ & $44(6)$ & 0.19 \\
\hline History of chronic kidney disease (1 year) & $13(5)$ & $17(2)$ & 0.18 \\
\hline Diabetes & $118(48)$ & $356(45)$ & 0.04 \\
\hline Residence in a long term care facility & $48(19)$ & $168(21)$ & 0.05 \\
\hline $\begin{array}{l}\text { Median (interquartile range) No of prescription drugs in } \\
\text { previous year }\end{array}$ & $17(14-22)$ & $16(12-20)$ & 0.34 \\
\hline \multicolumn{4}{|l|}{ Drug use in preceding 120 days: } \\
\hline Non-potassium sparing diuretics & $201(81)$ & $561(72)$ & 0.21 \\
\hline Potassium sparing diuretics $\dagger$ & $8(3)$ & $15(2)$ & 0.09 \\
\hline$\beta$ adrenergic receptor blockers & $111(45)$ & $303(39)$ & 0.12 \\
\hline Potassium supplements & $9(4)$ & $20(3)$ & 0.06 \\
\hline Non-steroidal anti-inflammatory drugs & $90(36)$ & $202(26)$ & 0.23 \\
\hline Renin-angiotensin-aldosterone inhibitors & $191(77)$ & $420(54)$ & 0.49 \\
\hline \multicolumn{4}{|l|}{ Fifth of income: } \\
\hline 1 (lowest) & $49(20)$ & $171(22)$ & 0.05 \\
\hline 2 & $64(26)$ & $167(21)$ & 0.11 \\
\hline 3 & $53(21)$ & $140(18)$ & 0.09 \\
\hline 4 & $41(17)$ & $159(20)$ & 0.1 \\
\hline 5 (highest) & $38(15)$ & $140(18)$ & 0.07 \\
\hline Missing & $\leq 5$ & $6(1)$ & 0.05 \\
\hline
\end{tabular}

${ }^{*}$ Difference between cases and controls divided by standard deviation; standardised differences $<0.1$ indicate good balance between cases and controls. †Amiloride and combination products; triamterene and combination products. 
Table 2| Association between hospital admission involving hyperkalaemia and recent antibiotic use

Use of antibiotic in preceding

14 days

TMP-SMX

Nitrofurantoin

Norfloxacin

Amoxicillin

TMP/SMX=trimethoprim-sulfamethoxazole.

*Adjusted for age category, congestive heart failure, chronic liver disease, chronic kidney disease, Charlson co-morbidity index, fifth of income, living in long term care facility, number of prescription drugs in previous year, number of years of spironolactone treatment, and drugs ( $\beta$ adrenergic receptor blockers, potassium sparing diuretics, non-potassium sparing diuretics, non-steroidal anti-inflammatory drugs, potassium supplements, renin-angiotensin-aldosterone inhibitors). 